\title{
Impact of Clinical Specialty on Attitudes Regarding Overuse of Inpatient Laboratory Testing
}

\author{
Deborah Korenstein, MD ${ }^{1,2 *}$, Solomon Husain, BS³, Renee L Gennarelli, MS², Cilian White ${ }^{4}$, \\ James N. Masciale, MA ${ }^{5}$, Benjamin R. Roman, MD, MSHP2,3
}

\begin{abstract}
${ }^{1}$ Memorial Sloan Kettering Cancer Center, Department of Medicine, New York, New York; ${ }^{2}$ Memorial Sloan Kettering Cancer Center, Center for Health Policy and Outcomes, New York, New York; ${ }^{3}$ Memorial Sloan Kettering Cancer Center, Department of Surgery, Head \& Neck Division, New York, New York; ${ }^{4}$ Morristown Medical Center, Department of Medicine, Morristown, New Jersey; ${ }^{5}$ Memorial Sloan Kettering Cancer Center, Department of Quality and Safety, New York, New York.
\end{abstract}

Routine laboratory testing is common among hospitalized patients, with associated harm. Attitudes toward testing and drivers across clinical specialties have not been described. We performed a cross-sectional study and anonymously surveyed inpatient clinicians (nurses, advanced practice providers, and physicians) at a tertiary cancer center regarding attitudes toward unnecessary laboratory testing and its drivers across clinical specialties. A total of 837 providers completed surveys (response rate $53 \%$ ). Most respondents agreed with daily testing of hospitalized patients and that daily labs generally enhance safety, and those from pediatric and surgical specialties generally valued testing less than others. Participants most commonly identified habit and institutional culture as important drivers of unnecessary testing. There were differences in other drivers across specialties, with pediatric clinicians identifying family pressure more commonly and fear of litigation less commonly compared to others. Future interventions to reduce unnecessary inpatient laboratory testing should acknowledge different attitudes based on specialty and tailor interventions accordingly. Journal of Hospital Medicine 2018;13:844-847. Published online first June 27, 2018. (C) 2018 Society of Hospital Medicine
$\mathrm{R}$ outine laboratory testing in hospitalized patients is common, with a high prevalence of unnecessary tests that do not contribute to patient management. ${ }^{1}$ Excessive laboratory testing of hospitalized patients can contribute to anemia ${ }^{2}$ and may cause patient discomfort, additional unnecessary testing resulting from false positive results, and higher out-of-pocket patient costs. Excessive testing can impact hospital budgets both directly (though direct costs are often low) and indirectly through costly downstream services and prolonged hospital stay. ${ }^{3}$ As part of the American Board of Internal Medicine (ABIM) Foundation's Choosing Wisely initiative, several professional societies have recommended against routine laboratory testing of hospitalized adult patients. ${ }^{4}$

Excessive inpatient laboratory testing has been documented mostly among adult internal medicine (IM) patients with studies of drivers of unnecessary testing and efforts to reduce it conducted in IM settings. ${ }^{5,6}$ Attitudes toward other issues related to testing overuse differ by specialty ${ }^{7}$ and are likely to similarly vary with regard to unnecessary laboratory testing. Understanding

\footnotetext{
*Address for correspondence: Deborah Korenstein, MD, Department of Medicine, Memorial Sloan Kettering Cancer Center, 485 Lexington Avenue, New York, NY 10017; Telephone: (646) 888-8147; Fax (929) 321-1518; E-mail: korenstd@mskcc.org

Additional Supporting Information may be found in the online version of this article.
}

Received: January 4, 2018; Revised: February 15, 2018;

Accepted: March 3, 2018

2018 Society of Hospital Medicine DOI 10.12788/jhm.2978 differences in attitudes by clinical specialty is critical for framing tailored approaches to reducing inappropriate care.

We performed a cross-sectional survey of a diverse group of hospital clinicians to describe attitudes and beliefs regarding laboratory testing and its overuse across clinical specialties (eg, medical, surgical, and pediatric). We hypothesized that attitudes toward the need for testing would differ across specialties.

\section{METHODS}

\section{Survey Development and Administration}

The study was conducted at Memorial Sloan Kettering Cancer Center, a tertiary academic cancer hospital in New York City. The 12-item survey was adopted from a previously administered but not formally validated survey (Online-only Appendix). ${ }^{5,8}$ The survey was pilot tested with four physicians, three NPs, two PAs, and three RNs and edited for content and clarity. All staff providers including NPs, PAs, RNs, and resident, fellow, and attending MDs working in the hospital during the two-week survey period (November 2-15, 2015) were eligible to participate and were emailed a link to the survey. The email invitation was resent three times during the survey period. Participants who completed the survey received a coupon for a free coffee. The study was reviewed by the Institutional Review Board and exempted from ongoing oversight.

\section{Measures}

Demographic items included clinical specialty, provider type, and gender (Appendix). The remaining survey questions included the following categories: 
TABLE 1. Respondent Demographics $(n=837)$

\begin{tabular}{lc}
\hline Characteristic & No. (\%) \\
\hline Female gender & $609(73)$ \\
\hline Clinical specialty & \\
\hline Medical or Med-Onc & $272(33)$ \\
Surgical & $236(28)$ \\
Pediatric & $114(14)$ \\
Other & \\
Critical care & $73(9)$ \\
$\quad$ Gynecologic & $46(6)$ \\
$\quad$ Neurologic & $39(5)$ \\
$\quad$ Other & $57(7)$ \\
\hline Provider type & \\
\hline Attending MD & $197(24)$ \\
Trainee MD & $122(15)$ \\
Fellow & $17(2)$ \\
Resident & \\
\hline APP & $108(13)$ \\
\hline NP & $46(6)$ \\
\hline PA & $347(41)$ \\
\hline RN & \\
\hline abercentages do not sum to 100 due to rounding & \\
oncologyiations: APP, advanced practice provider; MD, doctor of medicine; Med-Onc, medical & \\
\hline
\end{tabular}

1. Attitudes toward laboratory testing were evaluated by threeitems about accepted norms for lab testing and two items about fears (Table 2). Responses to these items used a four-point Likert scale (strongly agree to strongly disagree).

2. Drivers contributing to unnecessary testing were evaluated by presenting a list of possible contributing factors (Table 2). Responses to these items used a three-point Likert scale (contributes a lot, contributes a little, or does not contribute).

\section{Analysis}

We used univariate statistics to describe demographics and survey responses. We used the chi-square statistic to evaluate differences in attitudes and drivers by clinical specialty. We dichotomized responses regarding attitudes toward lab testing ("strongly agree" and "somewhat agree" vs "somewhat disagree" and "strongly disagree.") and beliefs regarding contributing drivers ("contributes a lot" vs all others). We grouped clinical specialty into medical/med-oncology, surgical, pediatric, and other (gynecological, critical care, and other).

We used logistic regression to explore the associations between attitudes/drivers and clinical specialty after adjusting for provider type, and report the overall $P$-value. We used pediatrics as the reference group to assess direct comparisons with each of the other specialties. We performed analyses with SAS statistical software, version 9.4 (SAS Institute, Cary, North Carolina) and considered $P<.05$ to be significant.

\section{RESULTS}

Among 1,580 eligible participants, 837 (53\%) completed surveys. Attending MD response rates ranged between $61 \%$ (surgical) to $86 \%$ (pediatric); rates were $59 \%$ for all trainees, $72 \%$ for PAs and $46 \%$ for RNs and NPs combined. Given privacy concerns, we were unable to collect detailed response rate information or any information about nonrespondents. The demographics are shown in Table 1.

\section{Attitudes toward Laboratory Testing}

The majority of respondents agreed that hospitalized patients should get daily labs (59\%), testing on the discharge day (52\%), and that daily testing generally enhances safety (55\%; Table 2). Fewer pediatric and surgical clinicians endorsed that laboratory testing should be done daily ( $56 \%$ and $47 \%$ respectively) and enhances patient safety ( $46 \%$ and $47 \%$ ). These differences were significant after adjusting for provider type. In addition, fewer pediatric providers endorsed the statement that daily laboratory testing helps avoid malpractice litigation. Overall, $68 \%$ of respondents agreed they would be comfortable with less testing.

\section{Drivers Contributing to Unnecessary Laboratory Testing}

The strongest drivers of unnecessary testing were seen as habit (94\% responding "contributes a lot") and institutional culture (89\% responding "contributes a lot"; Table 2). After adjusting for provider type, significant differences were observed based on clinical specialty. In particular, pediatric specialists were less likely to endorse fear of litigation $(P<.001)$ and more likely to endorse pressure from patient/family $(P=.0003)$ compared to all other specialties (Table 2, odd ratios not shown).

\section{DISCUSSION}

Overuse of laboratory testing in hospitalized patients is widely recognized in IM and likely to be prevalent in other clinical specialties. Our study elucidated differences in attitudes toward unnecessary testing and self-identified drivers across specialties in a diverse group of clinical providers at an academic cancer center. We found differences based on clinical specialty, with those caring for pediatric and surgical patients less likely than others to believe that testing should be done daily and that daily testing enhances patient safety. Furthermore, comfort with less testing was highest among pediatric specialists. Habit and institutional culture were recognized broadly as the strongest drivers of laboratory testing overuse.

Our findings regarding differences based on clinical specialty are novel. Respondents caring for pediatric patients generally placed lower value on testing, and IM clinicians were the most likely to endorse daily testing and to believe that it enhances patient safety and helps avoid malpractice litigation. The difference between adult and pediatric clinicians is surprising given the fundamental similarities between these specialties. ${ }^{9}$ Although some resource use studies have described differences across specialties, none has examined differences in laboratory testing or examined the practice patterns of clinicians 
TABLE 2. Attitudes toward Laboratory Testing and Drivers of Unnecessary Testing by Clinical Specialty

\begin{tabular}{|c|c|c|c|c|c|c|}
\hline Question & $\begin{array}{c}\text { All } \\
(n=837)\end{array}$ & $\begin{array}{l}\text { Pediatric } \\
(\mathrm{n}=114)\end{array}$ & $\begin{array}{l}\text { Medical/Med-Onc } \\
\quad(n=272)\end{array}$ & $\begin{array}{c}\text { Surgical } \\
(\mathrm{n}=236)\end{array}$ & $\begin{array}{c}\text { Other } \\
(\mathrm{n}=215)\end{array}$ & $P$ Value $^{\mathrm{a}}$ \\
\hline Attitudes toward testing & \multicolumn{6}{|c|}{ Strongly agree or somewhat agree, No (\%) } \\
\hline Hospitalized patients should have daily laboratory testing & $491(59)$ & $64(56)$ & $180(66)$ & $112(47)$ & $135(63)$ & $<.0001$ \\
\hline Hospitalized patients should have laboratory testing on discharge day & $434(52)$ & $77(68)$ & $158(58)$ & $76(32)^{b}$ & $123(57) b$ & $<.0001$ \\
\hline Daily laboratory testing generally helps avoid malpractice litigation & $400(48)$ & $40(35)$ & $152(56)^{b}$ & $98(42)$ & $110(51)$ & .0004 \\
\hline Asking for laboratory testing protects me from criticism & $281(34)$ & $31(27)$ & $105(39)^{b}$ & $68(29)$ & $77(36)$ & .0711 \\
\hline I would be comfortable if my hospitalized patients received LESS laboratory testing & $566(67)$ & $86(75)$ & $182(67)$ & $157(67)$ & $141(66)$ & .0821 \\
\hline Habit or training & 787 (94) & $112(98)$ & $259(95)$ & $215(91)^{b}$ & $201(93)$ & .0532 \\
\hline Ease of ordering & $683(82)$ & $102(89)$ & $223(82)^{b}$ & $180(76)^{b}$ & $178(83)$ & .0421 \\
\hline Discomfort with not knowing labs & $678(81)$ & $100(88)$ & $230(85)$ & $176(75)^{b}$ & $172(80)$ & .0110 \\
\hline Institutional culture & $748(89)$ & $107(94)$ & $248(91)$ & $197(83)^{b}$ & $196(91)$ & .0046 \\
\hline Concern that others will ask for data & $662(79)$ & $91(80)$ & $223(82)$ & $176(75)$ & $172(80)$ & .3458 \\
\hline Pressure from patient or family & $584(70)$ & $96(84)$ & $192(71)^{b}$ & $145(61)^{b}$ & $150(70)^{b}$ & .0003 \\
\hline
\end{tabular}

a $P$-values based on type III analysis of effects after adjusting for provider type

bSignificantly different odds as compared to pediatric

who are not physicians across specialties. ${ }^{10}$ Prior studies have documented the impact of training location on practice ${ }^{11,12}$, suggesting the importance of the local training culture. ${ }^{13}$ As physician personalities vary across clinical specialties ${ }^{14}$ it is likely that culture varies as well. Specialty-specific cultures are likely to strongly influence attitudes and practice patterns and warrant further exploration.

Clinicians in our sample identified drivers of unnecessary laboratory testing that were consistent with other studies, most frequently endorsing habit, followed by culture, discomfort with not knowing, and concern that someone will ask for the results. ${ }^{5,15}$ Previous studies have focused on IM and have not included nonphysicians or compared attitudes across specialties. We found that the largest differences in drivers by specialty were related to malpractice concerns and the perception of pressure from patients or families. The low endorsement of defensive medicine among clinicians serving pediatric populations may imply that interventions to reduce unnecessary care in hospitalized children may not need to address malpractice fear. In contrast, clinicians from pediatrics identified family pressure as a greater driver of unnecessary testing. Efforts to reduce unnecessary laboratory testing in pediatrics will need to address parent expectations.

Our findings have implications for efforts to reduce unnecessary testing. Culture, identified as a key driver of testing, reflects leadership priorities, institutional history, and other factors and is difficult to specifically target. Habit, the other most-endorsed driver, is a more promising target for quality improvement interventions, particularly those addressing care processes (eg, electronic ordering). Discomfort with not knowing and fear of being asked are drivers that might be influenced by better communication about information expectations by supervising physicians and hospital administration. Lastly, education about the potential harms of excessive testing may facilitate more targeted efforts to reduce testing overuse.

Our study has important limitations. The cancer focus of the center may have influenced provider attitudes and practices. Attitudes may differ at community centers, though important differences regarding routine laboratory testing are unlikely. Second, although our sample was large, our response rate was modest at 53\% and as low as $46 \%$ among RNs and NPs and we have no information regarding nonresponders. This response rate, though, was comparable to response rates seen in other large surveys. ${ }^{5,15}$ In addition, our results reflect clinician self-report; perceptions of necessity and the true need for testing may vary across specialties and the true subconscious drivers of behavior may differ. However, differences across specialties are likely to be valid even if there are other factors at play. Self assessment of unnecessary testing may also underestimate prevalence of the problem. Finally, our findings related to driv- 
ers of unnecessary testing are descriptive rather than quantitative given the lack of validated scales.

In conclusion, we evaluated attitudes toward routine laboratory testing in hospitalized patients in clinicians across specialties and found important differences. These findings speak to the diversity of cultures of medical care even within a single institution and point to the importance of studying attitudes about overused services across clinical specialties. In particular, as medical fields beyond IM increasingly recognize the importance of reducing medical overuse both in and out of the hospital, our findings highlight the importance of elucidating specialty-specific attitudes to optimize interventions to address unnecessary testing.

Disclosures: Mr. Husain, Ms. Gennarelli, Ms. White ${ }^{4}, M_{r}$. Masciale, MA ${ }^{5}$, and Dr. Roman, MD, have nothing to disclose. The work of Dr. Roman and Dr. Korenstein on this project was supported, in part, by a Cancer Center Support Grant from the National Cancer Institute to Memorial Sloan Kettering Cancer Center (P30 CA008748).

\section{References}

1. Zhi M, Ding EL, Theisen-Toupal J, Whelan J, Arnaout R. The landscape of inappropriate laboratory testing: a 15-year meta-analysis. PloS One. 2013;8(11):e78962. doi: 10.1371/journal.pone.0078962.

2. Thavendiranathan $P$, Bagai $A$, Ebidia A, Detsky AS, Choudhry NK. Do blood tests cause anemia in hospitalized patients? The effect of diagnostic phlebotomy on hemoglobin and hematocrit levels. J Gen Intern Med. 2005;20(6):520-524. doi: 10.1111/j.1525-1497.2005.0094.x.

3. Eaton KP, Levy $\mathrm{K}$, Soong $\mathrm{C}$, et al. Evidence-based guidelines to eliminate repetitive laboratory testing. JAMA Intern Med. 2017;177(12):1833-1839. doi: 10.1001/jamainternmed.2017.5152
4. Choosing wisely. http://www.choosingwisely.org/resources/. Accessed November 21, 2017.

5. Sedrak MS, Patel MS, Ziemba JB, et al. Residents' self-report on why they order perceived unnecessary inpatient laboratory tests. J Hosp Med. 2016;11(12):869-872. doi: 10.1002/jhm.2645.

6. Thakkar RN, Kim D, Knight AM, Riedel S, Vaidya D, Wright SM. Impact of an educational intervention on the frequency of daily blood test orders for hospitalized patients. Am J Clin Pathol. 2015;143(3):393-397. doi: 10.1309/ AJCPJS4EEM7UAUBV.

7. Sheeler RD, Mundell T, Hurst SA, et al. Self-reported rationing behavior among US physicians: a national survey. J Gen Intern Med. 2016;31(12):14441451. DOI: 10.1007/s11606-016-3756-5.

8. Roman BR, Yang A, Masciale J, Korenstein D. Association of attitudes regarding overuse of inpatient laboratory testing with health care provider type. JAMA Intern Med. 2017;177(8):1205-1207. DOI: 10.1001/jamainternmed.2017.1634.

9. Schatz IJ, Realini JP, Charney E. Family practice, internal medicine, and pediatrics as partners in the education of generalists. Acad Med. 1996;71(1):35-39.

10. Johnson RE, Freeborn DK, Mullooly JP. Physicians' use of laboratory, radiology, and drugs in a prepaid group practice HMO. Health Serv Res. 1985;20(5):525-547.

11. Chen C, Petterson S, Phillips R, Bazemore A, Mullan F. Spending patterns in region of residency training and subsequent expenditures for care provided by practicing physicians for Medicare beneficiaries. JAMA. Dec 10, 2014;312(22):2385-2393. doi: 10.1001/jama.2014.15973.

12. Sirovich BE, Lipner RS, Johnston M, Holmboe ES. The association between residency training and internists' ability to practice conservatively. JAMA Intern Med. 2014;174(10):1640-1648. doi: 10.1001/jamainternmed.2014.3337.

13. Smith $C D$, Korenstein D. Harnessing the power of peer pressure to reduce health care waste and improve clinical outcomes. Mayo Clin Proc. 2015;90(3):311-312. DOI: https://doi.org/10.1017/ice.2015.136

14. Vaidya NA, Sierles FS, Raida MD, Fakhoury FJ, Przybeck TR, Cloninger CR. Relationship between specialty choice and medical student temperament and character assessed with Cloninger Inventory. Teach Learn Med. 2004;16(2):150-156. doi: 10.1207/s15328015t/m1602_6

15. Studdert DM, Mello MM, Sage WM, et al. Defensive medicine among high-risk specialist physicians in a volatile malpractice environment. JAMA. 2005;293(21):2609-2617. doi: 10.1001/jama.293.21.2609 